\title{
Sophos: herramienta para medir la producción científica del Servizo Galego de Saúde.
}

\author{
Sophos: a tool to measure the scientific production \\ of "Servizo Galego de Saúde"
}

\begin{abstract}
Sobrido Prieto $\mathrm{M}^{1,2}$, Cabana Insua $\mathrm{T}^{1,3}$, González Guitián $\mathrm{C}^{1,2}$, Gutierrez Couto $\mathrm{U}^{1,4}$, Sanmartín Reinoso $\mathrm{Y}^{1,5}$, Pazo Martínez $\mathrm{RM}^{1,6}$, Delgado Rey $\mathrm{C}^{1,7}$, Osorio Calles $\mathrm{J}^{1,8}$, Calvo Ferrer $\mathrm{A}^{1}$, Perez Rodríguez $\mathrm{U}^{9}$, Cibeira Badía $\mathrm{M}^{1,5}$, Rodríguez Otero $\mathrm{C}^{1}$

${ }^{1}$ Bibliosaúde. Consellería de Sanidade. ${ }^{2 B i b l i o t e c a . ~ C o m p l e x o ~ H o s p i t a l a r i o ~ U n i v e r s i t a r i o ~ d e ~ A ~ C o r u n ̃ a . ~ S e r g a s . ~}{ }^{3 B}$ Biblioteca. Complexo Hospitalario Universitario de Santiago de Compostela. Sergas. ${ }^{4}$ Biblioteca. Complexo Hospitalario Universitario de Ferrol. Sergas. ${ }^{5}$ Biblioteca. Complexo Hospitalario Universitario de Vigo. Sergas. ${ }^{6}$ Biblioteca. Complexo Hospitalario Universitario de Ourense. Sergas. ${ }^{7}$ Biblioteca. Hospital da Costa. Sergas. ${ }^{8}$ Biblioteca. Complexo Hospitalario Universitario de Pontevedra. Sergas. ${ }^{9}$ Fundación Escola Galega de Administración Sanitaria
\end{abstract}

\section{Resumen}

En el artículo se describe la herramienta Sophos de ayuda a la investigación, creada para medir la actividad científica del Sistema Público de Saúde de Galicia. Describe su metodología de trabajo, realizada de forma cooperativa por todas las bibliotecas que componen Bibliosaúde, los informes que se obtienen con ella y las ventajas que resultan de sus estudios bibliométricos.

Palabras clave: Indicadores de Producción Científica; Publicaciones Científicas y Técnicas; Evaluación de la Investigación en Salud; Sistemas de Salud; Galicia.

\section{Introducción}

El desarrollo de la ciencia e innovación se consideran factores determinantes para asegurar el crecimiento económico y bienestar social de cualquier país. Este desarrollo científico se puede considerar como un balance inversión-beneficio. Las inversiones en ciencia se miden en términos de recursos financieros, gastos originados y recursos humanos disponibles. Los beneficios se evalúan a través de las publicaciones en revistas o los registros de patentes. Por ello, la publicación científica se convierte en un resultado importante y tangible de la investigación.

Los estudios bibliométricos tienen por objeto el tratamiento y análisis de las publicaciones científicas. Los resultados de estos estudios son de gran importancia, dadas las repercusiones sobre la asignación de fondos para la investigación e, incluso, la promoción profesional de los investigadores. En el ámbito sanitario español, los primeros estudios se publicaron en los años noventa ${ }^{1-4} y$ la década del $2000^{5-6}$. Si bien la evaluación de la actividad científica en Galicia tiene ciertos antecedentes, se trata de estudios parciales ${ }^{7}$, y de las memorias científicas de los hospitales del Servizo Galego de Saúde (Sergas) ${ }^{8-11}$.

En los últimos años el volumen de información y la necesidad de informes cada vez más completos ha generado la necesidad de herramientas que den soporte a la gestión de la investigación ${ }^{12}$.

Estas herramientas llevan en el mercado varios años. Se trata de bases de datos bibliográficas comerciales (In cites, elaborada por Thompson Reuters o Scival de Scopus), en ocasiones con limitaciones de cobertura.

\section{Abstract}

We describe Sophos platform, a software tool developed to measure the research activity produced by the Galician Public Health Service. Designed cooperatively by the libraries forming Bibliosaude, we detail its methodologic workflow and the useful bibliometric reports it provides.

Key words: Scientific Publication Indicators; Scientific and Technical Publications; Health Research Evaluation; Health Systems; Galicia

En el Sistema Público de Saúde de Galicia, la investigación, desarrollo e innovación, se encuentran integradas dentro de la estrategia de gestión del conocimiento, uno de los ejes de la Consellería de Sanidade en el que se hace necesaria la introducción de políticas de evaluación asociadas a la investigación como pieza fundamental de su gestión ${ }^{13}$. Para ello es necesario contar con fuentes de información sobre la actividad científica suficientemente fiables, y normalizadas para la posterior elaboración de indicadores bibliométricos. Para medir esta actividad, la Biblioteca Virtual do Sistema Público de Saúde de Galicia (Bibliosaúde) en colaboración con la Subdirección Xeral de Investigación, Docencia e Innovación ha desarrollado Sophos, una herramienta que permite medir la actividad científica del Sergas, que se enmarca dentro de la política de innovación de la Consellería de Sanidade.

La Consellería de Sanidade utiliza para la medición de la producción científica estándares de evaluación reconocidos ${ }^{14-15}$. Así, Sophos incluye solo aquellos artículos publicados y firmados por al menos un autor vinculado con el centro en la fecha de publicación, y en los que conste el nombre del centro de trabajo de la Consellería/Sergas.

\section{¿Cómo se elabora Sophos?}

Las etapas de elaboración de Sophos son:

1. Búsqueda bibliográfica: Para la localización de los artículos publicados anualmente se efectúa una búsqueda en las principales bases de datos tanto internacionales como nacionales (Tabla 1). 


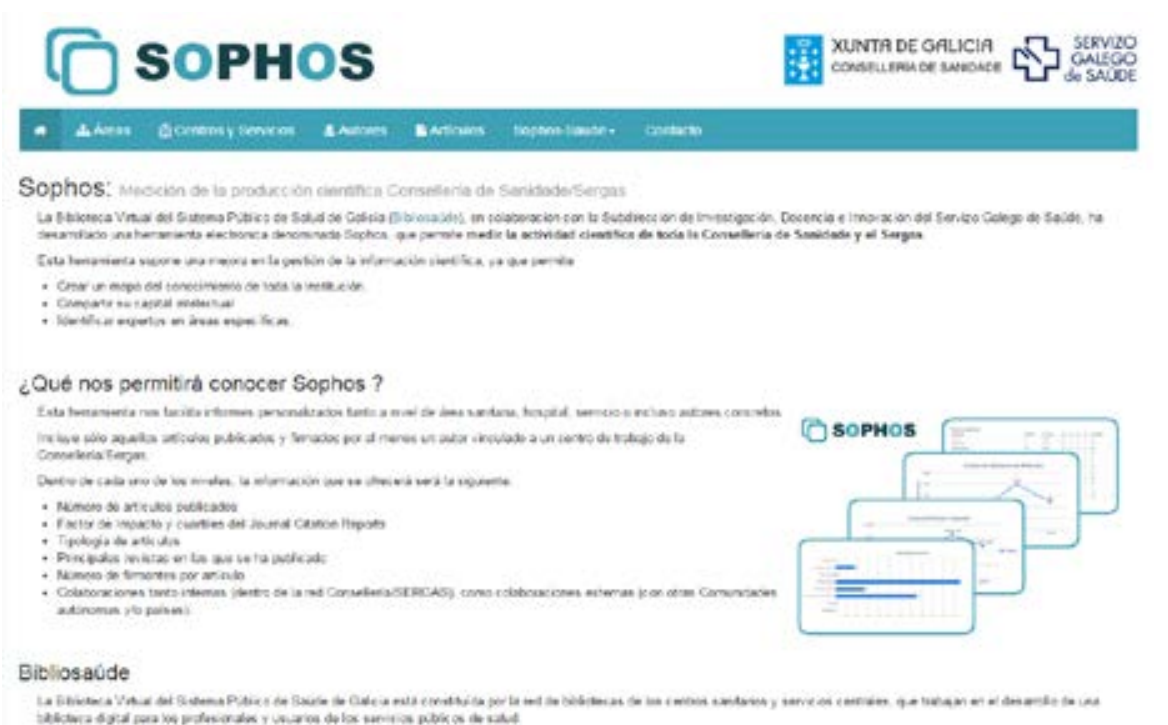

:Bibliosaúde

Gingation

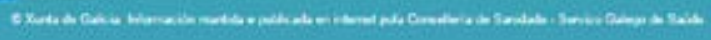

Tabla 1. Principales bases de datos de búsqueda en Sophos

\begin{tabular}{|c|c|}
\hline Internacionales & Nacionales \\
\hline Medline & CSIC \\
\hline Embase & Cuiden \\
\hline Web of Knowledge & Medes \\
\hline Scopus & IBECS \\
\hline
\end{tabular}

2. Incorporación de datos en Sophos y validación: El programa permite importar las referencias desde diferentes formatos normalizados (Ris, BibTeX...), tanto descargándolas directamente de las bases de datos, como a través de un gestor bibliográfico. Una vez depurados los duplicados e incorporados los resultados de la búsqueda, el personal técnico de Bibliosaúde lleva a cabo la validación de los registros individualmente, verifica las referencias y para dar un valor añadido incorpora los siguientes datos:

- Tipo de artículo. Se asignan las siguientes categorías: artículos de revisión, originales, de opinión y comunicaciones a congresos publicados en revistas científicas.

- Autores. Se incluyen los autores pertenecientes a cualquiera de los centros vinculados a la Consellería/ Sergas. Con el fin de eliminar posibles duplicados y evitar problemas de la variabilidad de la firma científica, los datos de los autores deben ser siempre verificados en el LDAP (Lightweight Directory Access Protocol) del Sergas.

- Sophos también permite incorporar los principales identificadores (ORCID, Google Scholar...) para facilitar la desambiguación de autores.
- Lugar de trabajo. Los artículos que se incorporan en Sophos se vinculan con el servicio y centro al que pertenecía el autor en el momento de su publicación. Para normalizar las diferentes denominaciones de un mismo servicio dentro de los centros de la Consellería / Sergas se ha creado un listado de los principales servicios.

- Colaboraciones. Con el fin de conocer las redes de colaboración existentes a nivel nacional y/o internacional, se indican las comunidades autónomas y/o países de centros que han participado, en calidad de autores, en cada uno de los estudios

3. Incorporación de factor de impacto: Anualmente se incorporan los datos del factor de impacto y cuartiles de las revistas según el Journal Citation Reports (JCR).

\section{¿Qué información ofrece Sophos?}

Esta herramienta facilita informes personalizados a nivel de área sanitaria, hospital, servicio o incluso autores concretos. Incluye solo aquellos artículos publicados y firmados por al menos un autor vinculado a un centro de trabajo de la Consellería/Sergas.

Dentro de cada uno de los niveles se ofrece información sobre el número de artículos publicados y sus tipos, el factor de impacto y cuartiles del JCR, principales revistas en las que se ha publicado, número de autores por artículo, número total de autores y redes de colaboración tanto internas (con otros centros y/o departamentos del Sergas) como externas (redes a nivel nacional e internacional). Además Sophos permite exportar los datos en formato.ris o.xls y añadirlos a aplicativos y plataformas que soporten estos formatos como gestores 
bibliográficos o al proyecto Curriculum Vitae Normalizado (CVN) de la FECYT ${ }^{16}$.

\section{Conclusiones}

Sophos ofrece información de interés a los diferentes actores del sistema sanitario. Para los gestores sanitarios (gerentes, directivos...) es un instrumento de planificación, seguimiento y evaluación de la investigación, permitiendo la identificación de grupos de excelencia, el análisis de la colaboración y la detección de áreas fuertes y aquellas que hay que reforzar en el sistema investigador de ciencias de la Salud de la Comunidad Gallega, además de información sobre resultados y repercusiones de la gestión I+D+l.

El personal investigador puede conocer su actividad, en qué revistas publica, el factor de impacto de sus publicaciones, cuartiles, posibles redes de colaboración con otros centros, así como otros criterios que miden la calidad de la investigación y de este modo poder realizar una autoevaluación y reutilización de esos datos en otras aplicaciones y plataformas.

\section{Bibliografía}

1. Terrada ML, López Piñero JM. La producción científica española y su posición en la Comunidad internacional. En: López Piñero JM (Coord). España-Ciencia. Madrid: Espasa Calpe; 1991. p. 73-109.

2. Camí J, Fernández MT, Gómez Caridad I. La producción científica española en Biomedicina y Salud. Un estudio a través del Science Citation Index (1986 1989). Med Clin (Barc) 1993; 101 (19): 72131

3. Espinosa de los Monteros J, et al. Una valoración de la investigación en biomedicina y salud en España. Madrid: Instituto de Estudios Sociales Avanzados; 1994

4. Espinosa de los Monteros J, Díaz V, Toribio A, Rodríguez Farré E, Larraga V, Conde J, Clavería LE, Muñoz E. La investigación biomédica en España. I: Evaluación del Fondo de Investigación Sanitaria (FIS) a través de los proyectos de investigación financiados en el período 1988-1995 a instituciones sanitarias asistenciales (hospitales) Med Clin (Barc). 1999 ;112(5):182-97.

5. Gómez Caridad I, Fernández Muñoz MT, Bordons Gangas M, Morillo Ariza F. La producción científica española en Medicina en los años 1994-1999. Rev Clin Esp. 2004; 204(2): 75-88.

6. Méndez-Vásqueza I, Suñén Pinyo E, Cervelló R, Camí J. Mapa bibliométrico de España 1996-2004: biomedicina y ciencias de la salud. Med Clin (Barc). 2008;130(7): 246-53.

7. Vázquez Montero J. Centro de saúde de Cambre, 1981-1996: Unha pequena análise bibliométrica. Cad Aten Prim. 1996; 3 (4): 196-201.

8. Xerencia de Xestión Integrada A Coruña. Memoria anual [Internet]. A Coruña: Servizo Galego de Saúde, Xerencia de Xestión Integrada A Coruña [Consultado 26 jul 2015]. Disponible en: http://hospitalcoruna.sergas.es/conocenos/Paginas/Memoria.aspx

9. Análisis de la producción científica del Complejo Hospitalario Universitario de Santiago y la Facultad de Medicina y Odontología durante el quinquenio 1990-1994. Santiago de Compostela: Complexo Hospitalario Universitario de Santiago; 1995.

10. Fundación Biomédica del Complexo Hospitalario Universitario de Vigo. Memorias anuales [Internet]. Vigo: Servicio Gallego de Salud, Fundación Biomédica del Complejo Hospitalario de Vigo. [Consultado 26 jul 2015]. Disponible en: http://chuvi.sergas.es/es/fundacionbiomedica/quienes_somos/Paginas/informe_anual.aspx

11. Área Sanitaria de Ferrol. Memoria 2010 [Internet]. Ferrol: Consellería de Sanidade, Servicio Galego de Saúde, Área Sanitaria de Ferrol; 2011. [Consultado 26 jul 2015] Disponible en: http://www.sergas.es/cas/Publicaciones/Docs/AtEspecializada/PDF-2116-es.pdf

12. Navarrete Cortés J, Banqueri Orzaez J. Los sistemas de información científica: herramientas para medir el impacto de la investigación biomédica. Med Clin (Barc). 2008; 131 (supl 5): 71-80.

13. Servizo Galego de Saúde. Estratexia Sergas 2014: la sanidad pública al servicio del paciente [Internet]. Santiago de Compostela: Consellería de Sanidade; 2011?. [Consultado 6 ago 2015]. Disponible en: http://www.sergas.es/Docs/Conselleria/ Estrategia_Sergas_2014.pdf

14. Moya-Anegón, F (dir.), Chinchilla Rodríguez, Z (coord.). Galicia: Patrones de colaboración científica (ISI-WOS, 2004). Santiago de Compostela: Xunta de Galicia; 2007

15. Moya-Anegón, F (dir), Chinchilla-Rodríguez Z (coord.), Corera-Álvarez E, Gómez-Crisóstomo R, González-Molina A, Muñoz-Fernández FJ, Vargas-Quesada B. Indicadores bibliométricos de la actividad científica española: 1990-2004. Madrid: Fundación Española para la Ciencia y la Tecnología; 2007

16. Fundación Española para la Ciencia y la Tecnología. Curriculum Vitae Normalizado CVN. [Internet]. Madrid: FECYT. [Consultada 8 ago 2015]. Disponible en: https:// cvn.fecyt.es/

Figura 2. Pantalla de búsqueda de sophos

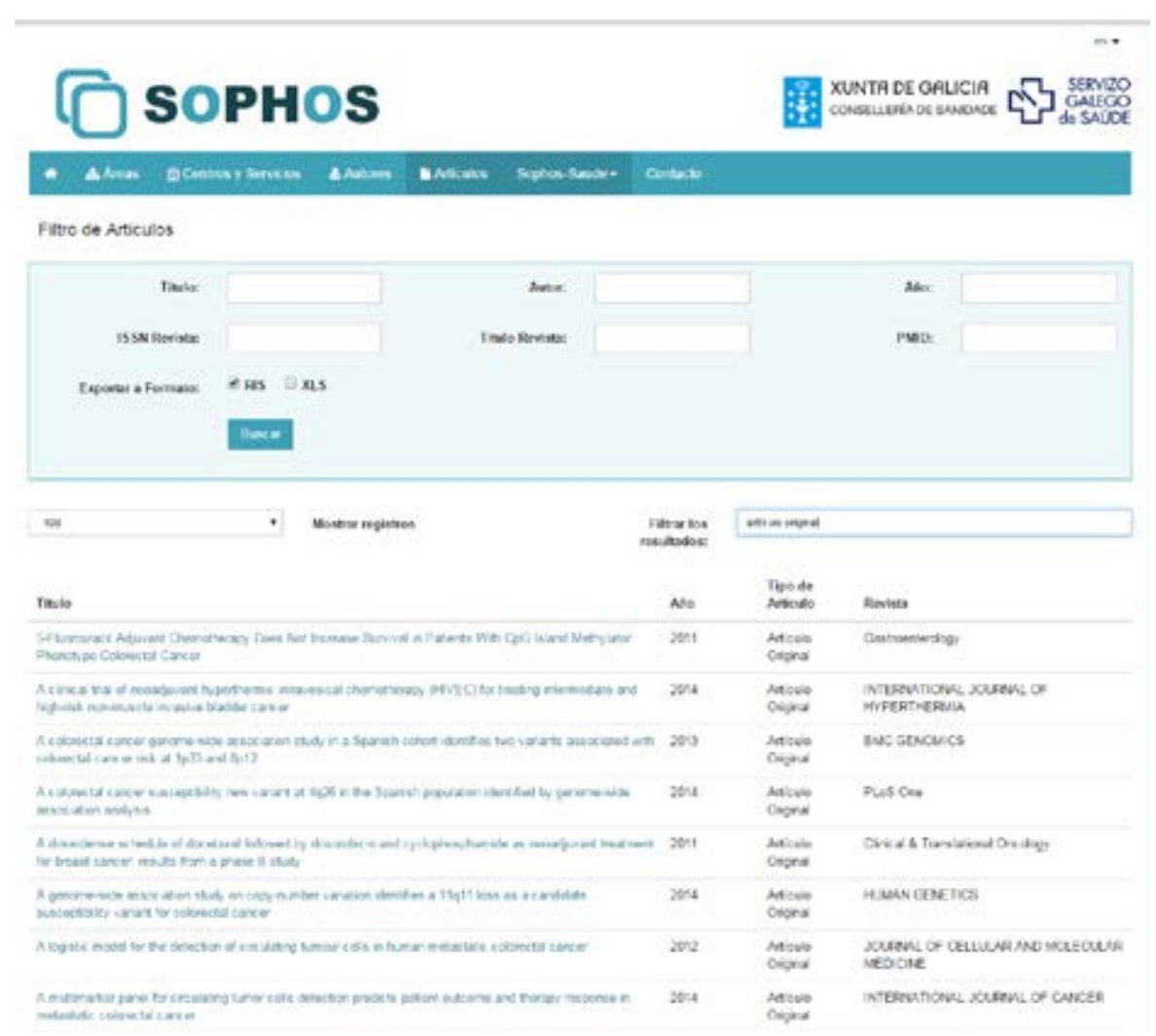

